\title{
Fast Interaction Trigger for the upgrade of the ALICE experiment at CERN: design and performance
}

\author{
Alla Maevskaya for the ALICE collaboration ${ }^{1, *}$ \\ ${ }^{1}$ Institute for Nuclear Research INR RAS, 117312, Moscow, Russia
}

\begin{abstract}
ALICE (A Large Ion Collider Experiment) at the CERN LHC is designed to study the properties of the Quark-Gluon Plasma (QGP) in heavy-ion collisions. In 2019-2020 the upgrade of the LHC will increase the luminosity and the collision rate beyond the design parameters of the current ALICE setup. To be able to benefit from the improved performance of the LHC, ALICE will upgrade several of its key detector systems including the Fast Interaction Trigger (FIT). FIT is designed to provide the functionality of the existing forward detectors while retaining or even improving their performance. It will provide minimum bias (MB) trigger with an efficiency higher than $90 \%$ for $\mathrm{pp}$ collisions, measure the luminosity for $\mathrm{pp}$ and $\mathrm{Pb}-\mathrm{Pb}$ collisions, and sustain interaction rates up to $1 \mathrm{MHz}$ and $50 \mathrm{kHz}$, respectively. FIT will determine the collision time with a resolution better than $50 \mathrm{ps}$ and will be used to measure the event multiplicity, the centrality, and the reaction plane. The detector consists of two arrays of Cherenkov radiators with micro-channel plate photo-multiplier (MCP-PMT) sensors, placed on both sides of the interaction point and of a single large-diameter scintillator ring. This article discusses the main design concepts, detector construction, beam test results, Monte Carlo simulations, and the results of detector performance studies.
\end{abstract}

\section{Introduction}

The ALICE experiment [1] at the CERN LHC is dedicated to the study of the Quark-Gluon Plasma - a hot and dense matter produced in ultra-relativistic heavy-ion collisions. After the LHC upgrade in 2019-2020, the interaction rate will reach $50 \mathrm{kHz}$ for $\mathrm{Pb}-\mathrm{Pb}$ and up to $2 \mathrm{MHz}$ for pp collisions. The physics program during the LHC Run 3 and 4 will take advantage of the unique properties of the upgraded ALICE detector and concentrate on heavy-flavor mesons and baryons, quarkonium states, low-mass dileptons up to very low $p_{\mathrm{T}}$, jets, and other rare processes. ALICE will be able to accumulate $10 \mathrm{nb}^{-1}$ of $\mathrm{Pb}-\mathrm{Pb}$ collisions, $6 \mathrm{nb}^{-1}$ of $\mathrm{pp}$ as well as $50 \mathrm{nb}^{-1}$ of $\mathrm{p}-\mathrm{Pb}$ collisions [2]. Data will be taken either with a Minimum Bias (MB) trigger or in a continuous mode. All events will be transmitted to the online systems. A new Fast Interaction Trigger detector (FIT) [3] will be installed.

The main components of the upgraded ALICE detector are shown in Fig. 1. FIT is required to generate trigger signals with a latency below $425 \mathrm{~ns}$, provide online luminosity measurements with a direct feedback to the LHC, reject beam-gas background events and determine forward multiplicity of charged particles. During the offline analysis, FIT data

*e-mail: alla@inr.ru 


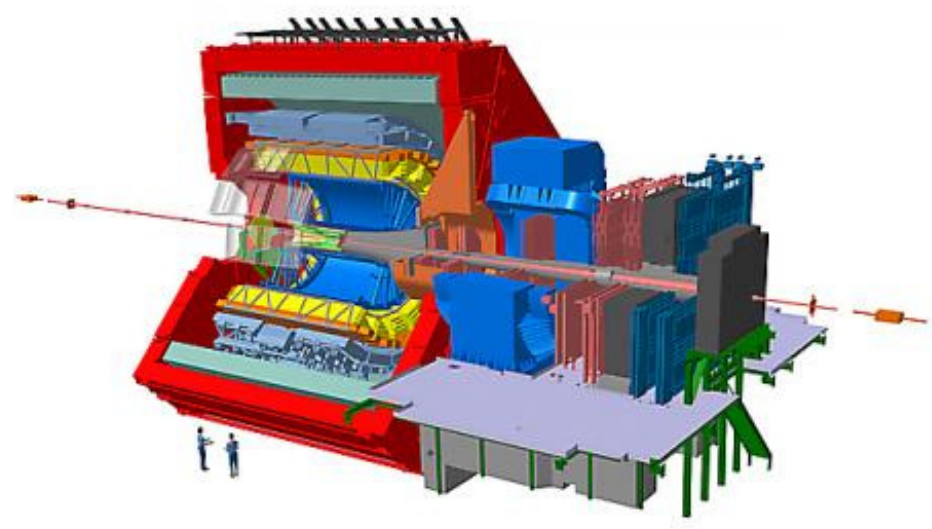

Figure 1. Schematic view of the upgraded ALICE detector

will provide centrality, event plane, and the collision time for Time-Of-Flight (TOF) based particle identification. The required time resolution is 50 ps or smaller for low multiplicity events.

\section{The Fast Interaction Trigger detector}

To satisfy the requirement of large acceptance and excellent timing, FIT will have a hybrid design. It will consist of two arrays of Cherenkov quartz radiators coupled to fast MCP-PMT photo sensors ( $\mathrm{T} 0 \mathrm{~A}+$ and $\mathrm{T} 0 \mathrm{C}+)$ and of a large segmented scintillator ring $(\mathrm{V} 0+)$, as shown in Fig. 2. The Cherenkov arrays will be placed around the beam pipe at $320 \mathrm{~cm}$ and -82 $\mathrm{cm}$ at the opposite sides of the interaction point (IP). Because of its proximity to the IP, to equalize the flight time and the effective quartz thickness, T0C+ will have a concave shape with a radius of $82 \mathrm{~cm}$ matching the distance to interaction point (IP). The TOA+ and T0C+ arrays will have 24 and 28 modules, respectively. Each T0+ module will consist of $2 \mathrm{~cm}$ thick quartz radiators coupled to a modified Planacon XP85012 MCP-PMT. The choice of Planacon XP85012 was based on its performance, compact size (28 mm thick) and a large surface of the photocathode $\left(53 \times 53 \mathrm{~mm}^{2}\right)$ with respect to the overall face area $\left(59 \times 59 \mathrm{~mm}^{2}\right)$ of the unit. The XP85012 MCP-PMT has a multi-anode structure ( $8 \times 8$ anodes array), which enables segmentation of up to 64 independent pixels. To match the division of the radiators into four equal elements, the MCP pixels are merged into four groups of 16 pads $(4 \mathrm{x} 4)$ each. In this way each $\mathrm{T} 0+$ module operates as four independent detector channels. The $\mathrm{V} 0+$ detector forms a flat disc with an outer diameter of $148 \mathrm{~cm}$ and an inner diameter of $8 \mathrm{~cm}-$ just sufficient to pass the beam line through. The active part of $\mathrm{V} 0+$ is made of a $4 \mathrm{~cm}$ thick EJ-204 plastic scintillators coupled to a grid of clear Asahi fibers transmitting the light to 2" Hamamatsu R5924-70 fine-mesh PMTs. The scintillator disc is divided into five rings and eight 45-degree sectors yielding a total of 40 detection elements.

\subsection{FIT electronics}

FIT trigger and readout electronics are being developed as a fully integrated system, based on an amplifier, a Constant Fraction Discriminator (CFD), on-board Time and Amplitude to Digital converters (TDC/ADC), FPGA processors, and GBT-based readout. The fact that the 

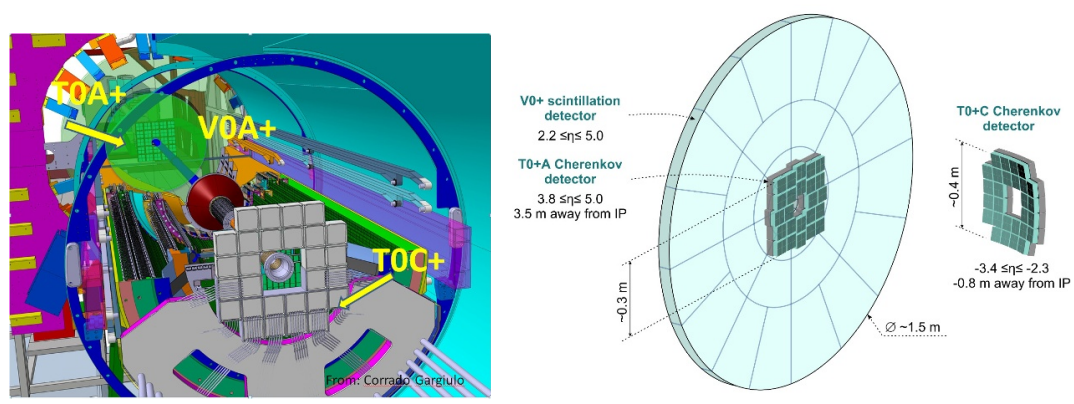

Figure 2. Left: View of FIT detector integrated in ALICE. Right: Active components of the FIT detector; the size and pseudorapidity coverage are indicated on the drawing

trigger decisions will be based on the digitized data (after TDCs \& ADCs) will allow for additional flexibility during the commissioning and operation of FIT.

\subsection{Test results}

$\mathrm{T} 0+$ prototypes were tested with a beam of pions and muons with momentum $p=6 \mathrm{GeV} / c$ at the CERN PS. A time resolution of around 33 ps was extracted including the signal deterioration along the $40 \mathrm{~m}$ of cables and the actual front-end analog electronics. Tests with a picosecond laser confirmed a linear response of the sensors within the required dynamic range of 1 to 500 particles per quadrant. According to simulations, the expected average particle load for $\mathrm{T} 0 \mathrm{~A}+$ modules the closest to the beam pipe in central $\mathrm{Pb}-\mathrm{Pb}$ collisions will be 280 but the distribution tails may reach up to 500 particles per quadrant.

\subsection{Aging tests}

During the planned six years of service the integrated anode current (IAC) accumulated by FIT T0A+ modules the closest to the beam pipe will be $\geq 0.57 \mathrm{C} / \mathrm{cm}^{2}$. To test the performance of the photosensor, a dedicated setup was used. Two quadrants of a Planacon XP85012 were illuminated in sub-saturated mode, while other two quadrants were shaded. After reaching an IAC of $0.5 \mathrm{C} / \mathrm{cm}^{2}$, the illuminated quadrant anodes lost $27 \%$ of the pulse amplitude. Such a relatively small drop is not problematic as it could be easily compensated by increasing the high voltage.

For a test in realistic environment a prototype of the FIT T0+ module was installed in the actual ALICE setup and operated there since the beginning of 2016. It yields a very stable time resolution and amplitude, and shows no sign of aging after 2.5 years of operation.

\subsection{FIT performance studies}

The main requirements concerning FIT are to reach a trigger efficiency of $90 \%$ or higher, and to maintain the centrality, event plane, and collision time resolution similar to those achieved during the LHC Run 1 and 2. To perform simulation studies, the detector geometry and performance parameters obtained during the prototype tests were included into the AliRoot [4] - ALICE framework for simulation, reconstruction, and data analysis. AliRoot takes into account all detectors, their support structures, magnetic field, and employs event generators to simulate collision systems. For pp interactions the Pythia 8 generator [5] was used and for 

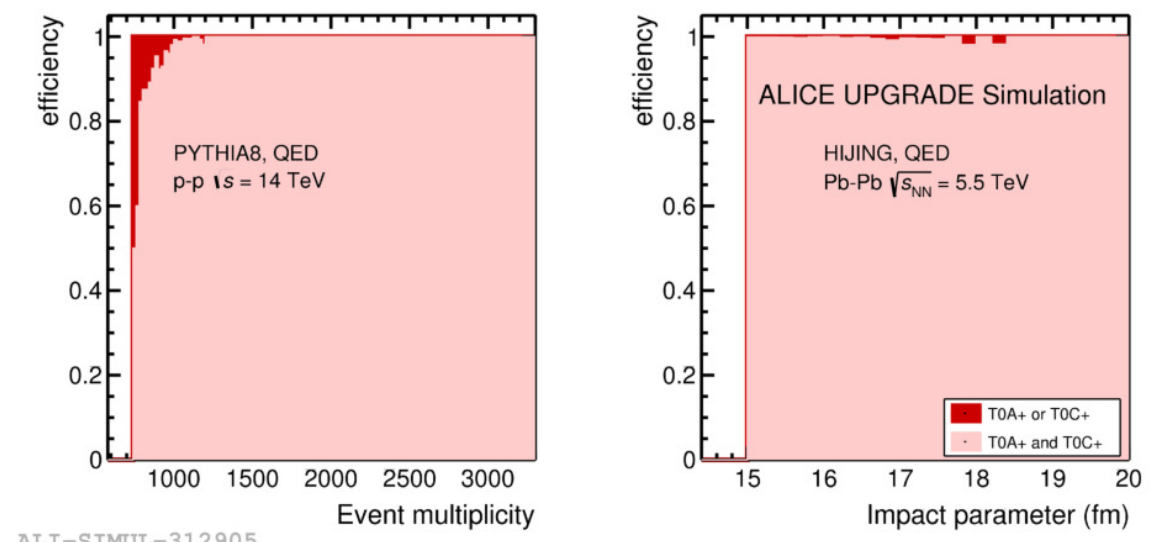

Figure 3. MB trigger efficiency measured by the FIT detector for $\mathrm{pp}$ (left) and $\mathrm{Pb}-\mathrm{Pb}$ (right) collisions

$\mathrm{Pb}-\mathrm{Pb}$ collisions - HIJING [6]. Electrons from electromagnetic interactions were added as an additional background. The left panel of Fig. 3 shows the registration efficiency for pp collisions as a function of event multiplicity. The right panel shows the efficiency as a function of impact parameter for a very peripheral $\mathrm{Pb}-\mathrm{Pb}$ collisions. The MB trigger conditions used for these calculations were generated as a coincidence between the $\mathrm{A}$ and $\mathrm{C}$ detectors and OR coincidence. The obtained efficiency was $98.8 \%$ for pp and $99.8 \%$ for peripheral $\mathrm{Pb}-\mathrm{Pb}$ collisions.
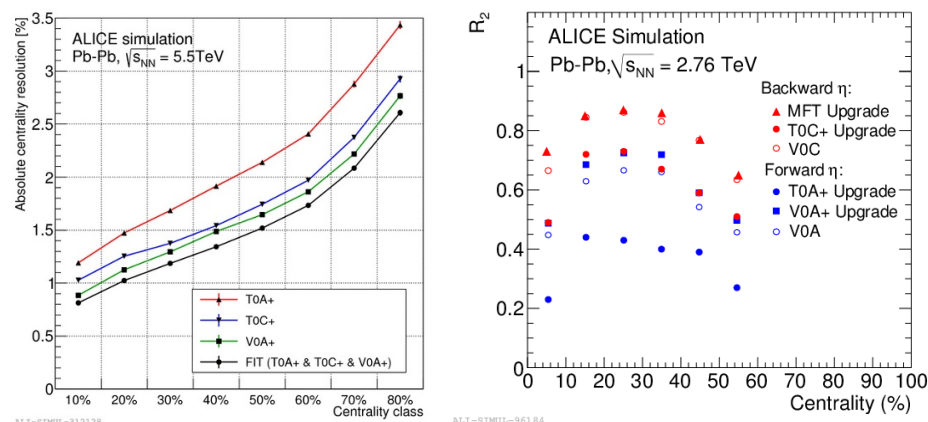

Figure 4. Left: Centrality resolution of FIT in the Run 3, Pb-Pb collisions at $\sqrt{s_{\mathrm{NN}}}=5.5 \mathrm{TeV}$. Right: FIT and MFT (upgrade) performance on event plane resolution R2

To extract the centrality resolution, $50000 \mathrm{~Pb}-\mathrm{Pb}$ collisions within a centrality range 0 $90 \%$ at $\sqrt{s_{\mathrm{NN}}}=5.5 \mathrm{TeV}$ were generated with HIJING. The left panel of Fig. 4 shows the centrality resolution for each component of FIT $-\mathrm{T} 0 \mathrm{~A}+$, T0C + and V0A+ and their combination. One could see that the centrality resolution obtained with the whole detector is below $1 \%$ for central events and increases to $2.5 \%$ for the most peripheral collisions. The event plane was estimated following the method of [7]. The generator included as inputs the 

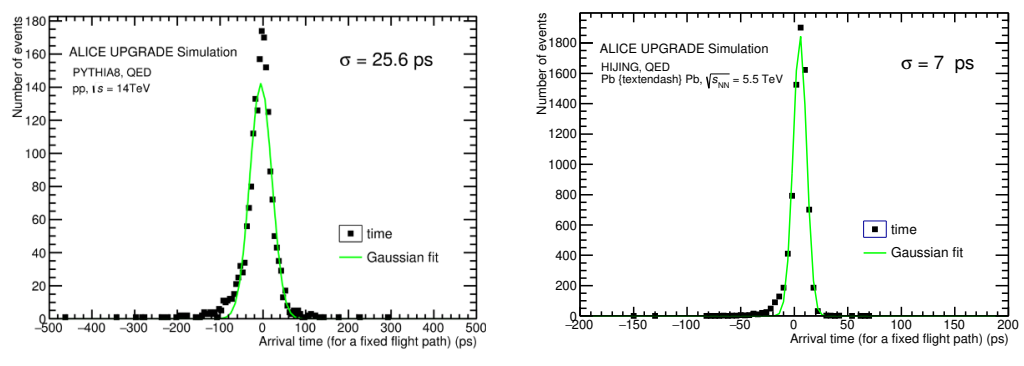

Figure 5. Collision time resolution of FIT in the Run 3 for pp collisions $\sqrt{s}=14 \mathrm{TeV}$ (left) and $\mathrm{Pb}-\mathrm{Pb}$ collisions, $\sqrt{s_{\mathrm{NN}}}=5.5 \mathrm{TeV}$ (right)

direct and elliptic flow distributions from the data. The right panel of Fig. 4 shows the event plane resolution for the current and upgraded forward detectors. The simulations demonstrate that the resolution obtained with FIT and Muon Forward Tracker (MFT) [8] detectors is equivalent to that of the current detectors.

The determination of the collision time with high precision is a very important part of the TOF-based particle identification. The collision time resolution is shown on the left panel of Fig. 5 for pp collisions at $\sqrt{s}=14 \mathrm{TeV}$ and on the right panel - for $\mathrm{Pb}-\mathrm{Pb}$ collisions at $\sqrt{s_{\mathrm{NN}}}=5.5 \mathrm{TeV}$ in the centrality range $0-90 \%$.

\section{Conclusions}

The strategy for the upgrade of the ALICE experiment is to benefit fully from the increased LHC luminosity in order to carry out measurements of rare probes requiring triggerless operation. To fulfill that goal, several elements of the setup had to be developed including the new Fast Interaction Trigger. FIT prototype tests at the CERN PS have demonstrated that the required functionality can be achieved. The time resolution and reliability of the new detector will be improved retaining the required efficiency, centrality, and event plane resolution. The conducted aging tests of the modified Planacon XP85012 MCP-PMTs predict only a modest $27 \%$ drop in the signal amplitude over the period of the Run 3. The sensor fulfills also the linearity requirements over the full amplitude range.

Acknowledgements. This work was supported for the INR RAS and National Research Nuclear University MEPhI participants within the Program of Russian groups activities in the ALICE upgrade by the Ministry of Education and Science of Russian Federation, contract No14.610.21.0003.

\section{References}

[1] ALICE Collaboration, Int. J. Mod. Phys. A 29, 1430044 (2014)

[2] ALICE Collaboration, J. Phys. G 41, 087001 (2014)

[3] W.H. Trzaska et al., NIM A 845, 463 (2017). doi:10.1016/j.nima.2016.06.029

[4] ALICE computing: Technical Design Report, CERN-LHCC-2005-018

[5] Torbjorn Sjostrand et al., Comput. Phys. Commun. 178, 852-867 (2008)

[6] Xin-Nian Wang et al., Phys. Rev. D 44, 3501-3516 (1991) 
[7] A. M. Poskanzer and S.A. Voloshin, Phys. Rev. C 58, 167 (1998)

[8] Technical Design Report for the Muon Forward Tracker, CERN-LHCC-2015-001, ALICE-TDR-018 